\title{
Shapes of a rising miscible droplet
}

\author{
Anatoliy Vorobev, ${ }^{1,}$ a) Timofey Zagvozkin, $^{2}$ and Tatyana Lyubimova ${ }^{2,3}$ \\ ${ }^{1)}$ Faculty of Engineering and Physical Sciences, University of Southampton, Southampton, UK, \\ SO17 1BJ \\ ${ }^{2)}$ Institute of Continuous Media Mechanics, Perm, Russia, 614013 \\ ${ }^{3)}$ Perm State University, Perm, Russia, 614990
}

(Dated: 7 January 2020)

We model the buoyancy-driven motion of a liquid droplet in an ambient liquid, assuming that the liquids are miscible. The classical representation of miscible liquids as a single-phase fluid with impurity (neglecting surface tension effects) cannot describe all experimental observations of moving droplets in a miscible environment, in particular, the tendency of droplets to pull to a spherical shape. In the framework of the classical approach, we show that the motion of a miscible droplet results in its instant dispersion (except for a very slow rise). We also model the motion of a miscible droplet in the framework of the phase-field approach, taking into account surface tension forces. We vary the value of the surface tension coefficient within a very wide range, modelling a droplet that rises preserving a spherical shape, or a droplet which dynamically becomes indistinguishable from the droplet with an interface endowed with no surface tension. We also show that by employing the concept of dynamic surface tension, one may reproduce the motion of a droplet that pulls into a sphere in the initial period of its evolution and that disintegrates similar to a droplet with zero surface tension at the later stages.

\section{INTRODUCTION}

We model the buoyancy-driven rise of a liquid droplet in a container filled with another liquid. We assume that the liquids are (slowly) miscible. Indeed, the diffusion coefficients in liquids are relatively low, $D \sim 10^{-9} \mathrm{~m}^{2} / \mathrm{s}$, so the complete dissolution of even a smaller droplet should take a while (the droplet with the radius $a_{0} \sim 10^{-3} \mathrm{~m}$ would dissolve over the period, $a_{0}^{2} / D \sim 10^{3} s$ ). During this period, the differences in the densities of two liquids in contact will engage a droplet into a hydrodynamic motion, which is the focus of the current work.

The buoyancy-driven motion of miscible droplets presents a great practical and theoretical interest. The interest in particular is motivated by the role of the surface tension forces in defining the shape and dynamics of a droplet moving in a miscible environment. There are many evidences that miscible interfaces should be endowed with some small dynamic surface tension (that may however disappear when the miscible system reaches the state of thermodynamic equilibrium). ${ }^{1,2}$ Such a conclusion can be even drawn from everyday observations, such as the dissolution of a honey droplet in tea (when a droplet of honey tends to have a spherical shape, the spherical shape can be only explained by the action of the surface tension forces). The values of the surface tension coefficient for a number of miscible interfaces were experimentally measured (see e.g. Refs. 3-7), with typically very low, but still different from zero, values (typically, in the order of $\sigma \sim 10^{-5} \mathrm{~N} / \mathrm{m}$ ). The most recent review on the dynamic surface tension can be found in work 8 . Nevertheless, accurate measurements of the low surface tension forces, and the correct application of the concept of the dynamic surface tension remains a topic of current active research work. For instance, one of the most recent experimental study, designed to measure the surface ten-

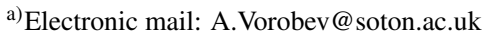

sion coefficient at a miscible (glycerol-water) interface by using the spinning drop tensiometry technique, turned out with the conclusion that the correct description of the dynamics of a miscible droplet in a spinning tensiometer does not require the use of the surface tension. ${ }^{9}$ The authors even state that the earlier measurements ${ }^{5,6}$ that were conducted with the use of the spinning drop tensiometer need revision.

The laboratory-based observations of falling and rising miscible inclusions in a container filled with another liquid are reported in e.g. papers 1 and 10. Based on the stability analysis, Kojima et al. ${ }^{10}$ demonstrated that the introduction of the surface tension force is needed for a rising droplet to remain spherical, as otherwise the spherical shape of a droplet is unstable to small perturbations, which eventually result in dispersion of the droplet in the ambient fluid. In addition, based on the qualitative comparisons of their theoretical and experimental results, Kojima et al. ${ }^{10}$ predicted 'the possible existence of a small time-dependent interfacial tension across the drop interface'. Later, the stability of a moving droplet was studied by other researchers, including Koh \& Leal ${ }^{11}$ and Pozrikidis $^{12}$, whose analysis (fulfilled for slowly rising immiscible droplets) indicated that the surface tension is capable of suppressing the growth of smaller perturbations, however, the perturbations with sufficiently large amplitude may still grow.

In this work, we conduct the direct numerical study of a rising miscible droplet with various levels of the surface tension forces, with the aim to understand when a moving miscible droplet may remain as a whole (not breaking and dispersing into the ambient fluid).

Our numerical study is carried with the use of two alternative approaches. First, we use the classical (traditional) representation for miscible liquids, modelling the hydrodynamic evolution of a mixture of two liquids on the basis of the hydrodynamic equations for a single-phase fluid. In the framework of the classical approach, the field of concentration is introduced in order to distinguish the components of a mixture and the hydrodynamic equations with addition of the equa- 
tion for the species transport are solved to trace the temporal changes in the system. Within this approach the interface is represented as a diffusive boundary with zero surface tension. The second approach of our work is the phase-field modelling that, in particular, adds the surface tension effects even when tracing the dynamics of miscible boundaries.

The theoretical approach of the current study was earlier used for modelling of the dynamics of a miscible droplet in a spinning drop tensiometer, ${ }^{13}$ the development of the Rayleigh-Taylor, Kelvin-Helmhotz and Holmboe instabilities at a miscible interface, ${ }^{14,15}$ the displacement of one liquid by another from a capillary tube, ${ }^{16}$ the development of frozen waves at a miscible interface, ${ }^{17,18}$ and others.

The phase-field approach was also used (and validated) for modelling the buoyancy-driven motion of immiscible inclusions ${ }^{19,20}$. The rise of a miscible inclusion was the focus of our earlier study ${ }^{21}$, where we examined the interplay of the droplet's motion and the interfacial diffusion. We studied the rise of near-spherical droplets that slowly loose some material due to interfacial diffusion. The key focus of the current work is the effect of the surface tension forces on the droplet's shape. We want to understand the dynamics of droplets with lower surface tension coefficients, when droplets would tend to disperse into smaller droplets rather than to rise as a whole.

\section{PHYSICAL MODEL}

\section{A. Classical approach}

Within the classical description, the mixture of two miscible liquids is represented as a single-phase fluid with impurity. To trace the presence of the second liquid in a mixture the field of concentration is introduced. The concentration is first defined as the mass fraction of the second component in the mixture, but in order to simplify comparisons with the results of the phase-field modelling, we also shift the reference point for the concentration field, $C \rightarrow(C-1 / 2)$, so $C=-1 / 2$ corresponds to the pure first component and $C=1 / 2$ is the pure second component. We assume that mixture remains isothermal during the evolution (the latent heat that may be associated with the dissolution/absorption process is neglected) and we also assume that both components of the mixture are incompressible. Nevertheless, the density of the mixture remains a function of concentration, which is set by a simple linear approximation,

$$
\rho=\rho_{1}\left(1+\phi\left(C-\frac{1}{2}\right)\right)
$$

Here $\rho_{1}$ and $\rho_{2}$ are the densities of the mixture components, and $\phi=\left(\rho_{2}-\rho_{1}\right) / \rho_{1}<1$ is the density contrast.

The evolution of a mixture is determined by the equations that reflect the laws of conservation of momentum, species, and mass,

$$
\frac{\partial \vec{u}}{\partial t}+(\vec{u} \cdot \nabla) \vec{u}=-\nabla \frac{\Pi}{\rho_{1}}+\frac{\eta}{\rho_{1}} \nabla^{2} \vec{u}+\phi C \vec{g},
$$

$$
\frac{\partial C}{\partial t}+(\vec{u} \cdot \nabla) C=D \nabla^{2} C
$$

$$
\nabla \cdot \vec{u}=0
$$

One may recognise that these are the equations for natural convection written in the framework of the Boussinesq approximation. ${ }^{22}$ These equations adopt the following notations: $\vec{u}$ is the velocity vector, $\Pi$ is the pressure, $\vec{g}$ is the gravity acceleration, $t$ is the time, $\eta$ is the viscosity coefficient, and $D$ is the diffusion coefficient. For simplicity, it is assumed that both $\eta$ and $D$ are constants.

Next, we non-dimensionalise the governing equations (2)(4) by using the following scales for the length, velocity, time, and pressure,

$$
L_{*}=R, u_{*}=\frac{\eta}{\rho_{1} L_{*}}, \tau=\frac{\rho_{1} L_{*}^{2}}{\eta}, p_{*}=\rho_{1} u_{*}^{2} .
$$

Here $R$ is the radius of a container.

The governing equations written in the non-dimensional form read as follows

$$
\frac{\partial \vec{u}}{\partial t}+(\vec{u} \cdot \nabla) \vec{u}=-\nabla \Pi+\nabla^{2} \vec{u}-G r \vec{\gamma},
$$

$$
\frac{\partial C}{\partial t}+(\vec{u} \cdot \nabla) C=\frac{1}{S c} \nabla^{2} C
$$

$$
\nabla \cdot \vec{u}=0
$$

Here $\vec{\gamma}$ is the unit vector directed upwards, $\vec{\gamma}=-\vec{g} / g$.

These equations include two non-dimensional parameters, which are the Grashof and Schmidt numbers,

$$
G r=\frac{\rho_{1}^{2} \phi g L_{*}^{3}}{\eta^{2}}, S c=\frac{\eta}{\rho_{1} D} .
$$

\section{B. Phase-field approach}

To take into account the surface tension effects at the interface we describe the evolution of a mixture in the framework of the phase-field approach, adopting the Boussinesq approximation of the full Cahn-Hilliard-Navier-Stokes equations. ${ }^{17,23}$ In the dimensional form, the governing equations read

$$
\begin{gathered}
\frac{\partial \vec{u}}{\partial t}+(\vec{u} \cdot \nabla) \vec{u}=-\nabla \frac{\Pi}{\rho_{1}}+\frac{\eta}{\rho_{1}} \nabla^{2} \vec{u}-C \nabla \mu \\
\frac{\partial C}{\partial t}+(\vec{u} \cdot \nabla) C=\frac{\alpha}{\rho_{1}} \nabla^{2} \mu \\
\nabla \cdot \vec{u}=0 .
\end{gathered}
$$

$$
\mu=-\phi(\vec{g} \cdot \vec{r})+2 a_{T} C+4 b C^{3}-\varepsilon \nabla^{2} C .
$$


Here $\mu$ is the chemical potential, $\alpha$ is the mobility coefficient, $a_{T}$ and $b$ are the parameters that define the thermodynamic model of the binary mixture, $\varepsilon$ is the capillary constant, and $\vec{r}$ is the radius-vector.

The equation for the momentum conservation (10) includes the term that is called the Korteweg force. This term describes the effect of the surface tension on the morphology of the interface. It also describes the generation of the flows along the interface due to spatial variations of the interfacial stresses (the Marangoni effect). In our equations, this term also includes the gravity force, which can be easily understood by looking at the equation for the chemical potential (13).

From the equation for the species transport (11), one may understand that in the phase-field approach the diffusion flux is determined by the extended Fick's law, i.e. not through the gradient of the concentration (as in (3)) but through the gradient of the chemical potential. ${ }^{22}$ The thermodynamic equilibrium is determined by a constant value of the chemical potential, and the diffusion is induced when the chemical potential becomes spatially inhomogeneous. This description is particularly relevant for partially miscible liquids, the offequilibrium mixtures for which the state of thermodynamic equilibrium is heterogeneous. For instance, the equilibrium states of many binary mixtures are defined by phase diagrams with upper critical consolute points. For such mixtures, a temperature quench shifts a binary system off equilibrium. If however the new temperature of the mixture remains below the consolute point, then the new state of thermodynamic equilibrium may remain heterogeneous (which is also determined by the mass balance, - a smaller droplet may completely dissolve, although a larger droplet may shrink in size until the state of thermodynamic equilibrium is reached). The intensity of the diffusion process is determined by the mobility coefficient $\alpha$, which value can be correlated with the classical diffusion coefficient by the relation, $D=\alpha \frac{d \mu}{d C} / \rho_{1}$.

One sees that the definition of the chemical potential (13) includes the gravity term, two terms that define the classical part of the chemical potential, and the term that is determined by the capillary effect.

The amplitude of the capillary term is determined by the capillary constant, $\varepsilon$ that is assumed to be so small that the capillary term can be assumed negligible everywhere expect for the zones of strong inhomogeneities in the field of concentration, which correspond to interfaces. The capillary term in the chemical potential determines two very different physical effects: the shape of the interfacial boundary and the rate of interfacial diffusion. Indeed, disparity of intermolecular forces of two liquids in contact explains the existence of the surface tension effect. The same disparity also explains the existence of the potential barrier at the interface that needs to be overcome by the molecules in order for the molecules to diffuse from one phase to another.

For the classical part of the chemical potential (13) we use the Landau free energy function, ${ }^{24}$

$$
f_{0}=a_{T} C^{2}+b C^{4}, \quad \mu_{0}=\frac{d f_{0}}{d C}=2 a_{T} C+4 b C^{3} .
$$

The Landau free energy function is originally written for a near-critical system, with two phenomenological coefficients: $a_{T} \sim\left(T-T_{c}\right)$ that can be of any sign and $b$ that is always positive (here $T$ and $T_{c}$ are the temperature and the critical temperature, respectively). We however use the Landau function to describe arbitrary states of a binary system, not compulsory near the critical (consolute) points. The coefficients $a_{T}$ and $b$ are the two phenomenological parameters that can be determined by fitting the shape of the phase diagram of a particular binary mixture. Although, we still assume that $a_{T}$ can be of any sign, and $b$ is just positive.

Expression (14) is particularly convenient for description of the liquid/liquid binary mixtures with an upper critical (consolute) point, which are miscible in all proportions if the mixture temperature is above the critical point, and which are only partially miscible if the mixture temperature is below the critical point. This is the most common shape of a phase diagram for liquid/liquid binary mixtures. This phase behaviour is also typical for 'immiscible alloys', such as sulfides and silicate systems, glasses, metallic composites used as superconductors, magnetic materials, etc. ${ }^{25,26}$

As already noted, the first term of the chemical potential determines the gravity force, as substitution of this term into the Navier-Stokes equation (10) gives a standard expression for the gravity force, $-C \nabla \mu \rightarrow \phi C \vec{g}$. In addition, the first term of the chemical potential determines the effect of barodiffusion, ${ }^{22,27 ?, 28}$ which, in our case, is the preferential diffusion of heavier molecules downwards and, correspondingly, the preferential diffusion of lighter molecules upwards.

Indeed, the state of thermodynamic equilibrium of a binary mixture is determined by

$$
\vec{u}=0, \quad \Pi=0, \quad \mu=0,
$$

and the equilibrium concentration profile is determined by the equation,

$$
-\phi(\vec{g} \cdot \vec{r})+2 a_{T} C+4 b C^{3}-\varepsilon \nabla^{2} C=0 .
$$

By solving this equation (e.g. by assuming that in the state of equilibrium the concentration is a function of one (vertical) coordinate) one may easily see that the first term in equation (16) sets the gravitational stratification in the concentration profile.

One may also notice that the substitution of the expression for the chemical potential into the equation for the species transport cancels the gravity term. Nevertheless, the effect of barodiffusion still influences the concentration field through the boundary conditions imposed at the horizontal walls. Indeed, to exclude the diffusive transport through the wall, one needs to say that the normal derivative of the chemical potential is zero at the wall, which involves the gravity term thus influencing solutions for the concentration profiles.

To non-dimensionalise the governing equations (10)-(13), the following typical scales are chosen,

$$
L_{*}=R, u_{*}=\frac{\eta}{\rho_{1} L_{*}}, \tau=\frac{\rho_{1} L_{*}^{2}}{\eta}, \Pi_{*}=\rho_{1} u_{*}^{2}, \mu_{*}=b .
$$

Then, the governing equations in the non-dimensional form 
read

$$
\begin{gathered}
\frac{\partial \vec{u}}{\partial t}+(\vec{u} \cdot \nabla) \vec{u}=-\nabla \frac{\Pi}{\rho}+\frac{\eta}{\rho} \nabla^{2} \vec{u}-\frac{1}{M} C \nabla \mu, \\
\frac{\partial C}{\partial t}+(\vec{u} \cdot \nabla) C=\frac{1}{S c} \nabla^{2} \mu, \\
\nabla \cdot \vec{u}=0 .
\end{gathered}
$$$$
\mu=\operatorname{GrM}(\vec{\gamma} \cdot \vec{r})+2 A C+4 C^{3}-C n \nabla^{2} C
$$

These equations include several non-dimensional parameters. These are the Grashof and Schmidt numbers,

$$
G r=\frac{\rho_{1}^{2} \phi g L_{*}^{3}}{\eta^{2}}, S c=\frac{\eta}{\alpha \mu_{*}} .
$$

The definition of the Grashof number coincides with the corresponding parameter (9) that was introduced in the subsection that sets the classical approach. The definition of the Schmidt number is different by involvement of the mobility coefficient $\alpha$ instead of the standard diffusion coefficient $D$. We still treat this parameter as the Schmidt number, using the same notation, assuming that the diffusion coefficient in the phase-field theory, can be defined as $D=\alpha \mu_{*} / \rho_{1}$. In addition, similarly to the classical approach, we assume that the viscosity coefficient $\eta$ is constant (one value for both components).

Next, the thermodynamic model of the mixture, is defined by an additional non-dimensional parameter, that can be interpreted as the 'mixture temperature',

$$
A=-\frac{a}{\mu_{*}} .
$$

The strength of the capillary effects is determined by two parameters, the Cahn and Mach numbers,

$$
C n=\frac{\varepsilon}{\mu_{*} L_{*}^{2}}, M=\frac{u_{*}^{2}}{\mu_{*}}=\left(\frac{\eta_{*}}{\rho_{*} L_{*}}\right)^{2} \frac{1}{\mu_{*}} .
$$

The Cahn number also defines the thickness of the interface. For instance, the equilibrium thickness of a flat interface (neglecting the gravity effects) is $\delta_{0}=\sqrt{-C n / A}$. The Mach number determines the amplitude of the Korteweg force: the capillary effects are insignificant at higher values of the Mach number, so equation (10) transforms into equation (2); and the capillary effects are important at lower values of the Mach number.

As already noted, the benefit of the phase-field approach is a possibility to take into account the surface tension effects. The coefficient of the surface tension can be determined from the concentration field, as

$$
\sigma=\frac{E_{i}}{S}=\frac{C n}{M S} \int_{V}(\nabla C)^{2} d V
$$

Here $E_{i}$ is the interfacial energy (scaled by $\rho_{1} u_{*}^{2} L^{3}$ ) and $S$ is the surface area of a droplet. The coefficient of the surface tension is given in the units of $\rho_{1} u_{*}^{2} L_{*}$.
The modulus of the concentration gradient is close to zero almost everywhere except the interface. At the interface, the value of the concentration gradient can be estimated as $|\nabla C| \sim$ $1 / \delta$ (where $\delta$ is the current interface thickness), and thus the value of the surface tension coefficient can be estimated as

$$
\sigma \sim \frac{C n}{M S} \frac{S \delta}{\delta^{2}}=\frac{C n}{M \delta}
$$

\section{PROBLEM STATEMENT}

We model the buoyancy-driven rise of a small single droplet in a container filled with another liquid. We assume that the container has the shape of a cylinder with the circular cross section. To lessen the influence of the walls of the container on the droplet's behaviour, we assume that the droplet's radius is considerably smaller than the container's radius. In addition, we consider a sufficiently tall container, which allows us to neglect the influence of the bottom and top boundaries on the droplet's dynamics.

We solve the $2 D$ problem assuming the axial symmetry. The cylindrical system of coordinates is adopted, with the radial and vertical coordinates denoted by $r$ and $z$, respectively.

At the initial moment, we assume that the droplet is motionless, there is no fluid motion in the container, the droplet has a spherical shape, and the droplet is positioned at the centreline at the height of $z_{0}$. In other words, the initial velocity and concentration fields are set by the following expressions,

$$
\vec{u}_{0}=0, C_{0}=\frac{1}{2} \tanh \left(\frac{\sqrt{r+\left(z-z_{0}\right)^{2}}-r_{0}}{\delta_{0}}\right) .
$$

In our current work, the initial position of the droplet is always set by $z_{0}=0.5$, the initial radius of the droplet is set by $r_{0}=0.1$, and the initial thickness of the liquid/liquid interfacial boundary is $\delta_{0} \approx 0.014$ (which is the equilibrium thickness for $A=-0.5$ and $C n=10^{-4}$ ).

The governing equations formulated in section II are also supplemented with the boundary conditions. At the lower wall of the container we set,

$$
z=0: u_{r}=u_{z}=0, \frac{\partial C}{\partial z}=0, \frac{\partial \mu}{\partial z}=0
$$

At the upper wall,

$$
z=H: u_{r}=u_{z}=0, \frac{\partial C}{\partial z}=0, \frac{\partial \mu}{\partial z}=0 .
$$

At the centreline,

$$
r=0: u_{r}=0, \frac{\partial u_{z}}{\partial r}=0, \frac{\partial C}{\partial r}=0, \frac{\partial \mu}{\partial r}=0 .
$$

At the side walls,

$$
r=1: u_{r}=u_{z}=0, \frac{\partial C}{\partial r}=0, \frac{\partial \mu}{\partial r}=0 .
$$

These boundary conditions are written for the phase-field approach II B. The phase-field equations are of the higher 
(fourth) order in terms of the concentration field, and they require one additional condition for the concentration field. To exclude the diffusion flux we set the boundary condition on the chemical potential, and the second condition on the concentration determines the wetting properties at the walls. In this work, we are interested in the dynamics of the droplet far from the walls, which allows us to accept the neutral wetting conditions, when the contact line is just orthogonal to the wall.

The boundary conditions for the classical approach II A are exactly the same, with the only difference that those equations do not include the chemical potential, and hence the conditions for the chemical potential are not needed (it is not possible to set the wetting properties in the classical model, and the conditions for the concentrations would reflect no-diffusion through the walls).

\section{NUMERICAL APPROACH}

The equations are solved in terms of vorticitystreamfunction using the finite-difference approach, adopting the second-order formulae for spatial derivatives and the first-order formulae for time derivatives. The equation for the stream-function is solved by Gauss-Seidel method. The spatial discretisation is uniform. An explicit marching scheme with a constant time step is implemented.

Figure 1 depicts the numerical results that are obtained with the use of the grids with the different number of the grid points. One may observe the convergence of our results, indicating that the grid with $450 \times 2700$ grid-nodes is sufficiently good to obtain the accurate results. The requirements on numerical resolution are stricter for the phase-field approach, and become stricter for lower values of the Mach number, lower Cahn numbers, and higher Grashof numbers. Figure 1 shows the results for one of the most challenging calculations of this work.

All calculations are fulfilled for the height of the computational domain $L=6$. We found that this height is sufficient for a (near-spherical) droplet to reach the state of the rise with the constant (terminal) velocity, and for a dispersing droplet to undergo all dispersion stages. We also found that until the droplet is really close to the upper wall $(z>5.5)$ the influence of the upper wall on the droplet's motion remains insignificant.

\section{ESTIMATION OF PARAMETERS}

Let us now estimate the values of the non-dimensional parameters. The experiments 1 and 10 deal with the corn syrupwater, glycerol-water mixture, and molasses-glycerol mixtures. These mixtures are characterised by higher contrasts in viscosity coefficients. Our interest however is motivated by experiments with the weak (very diluted) water-based mixtures, similar to the ones used in work 29, when the physical parameters of the mixture components are very close to each other.

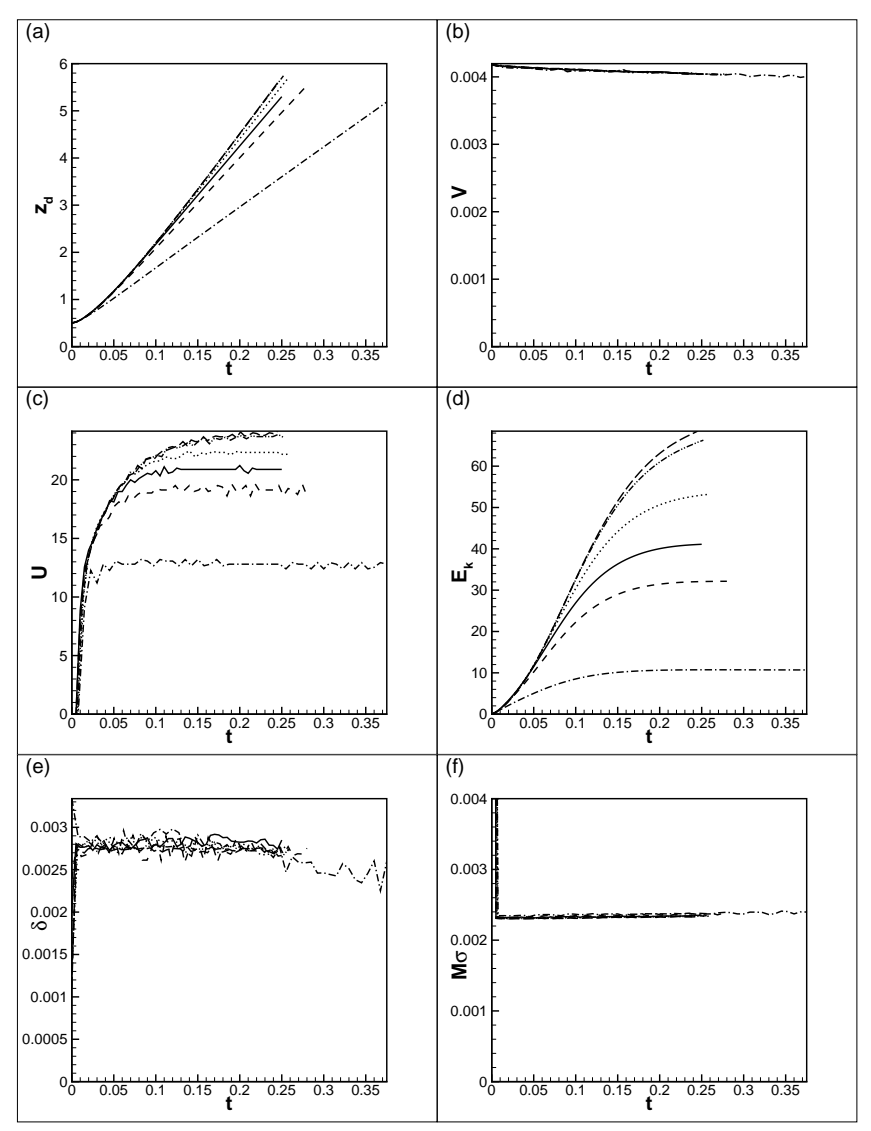

FIG. 1. The integral parameters of a rising droplet calculated with the use of different numerical grids: $250 \times 1500$ - dash-dot lines, $350 \times$ 2100 - dashed line, $450 \times 2700$ - solid lines, $550 \times 3300$ - dotted lines, $650 \times 3900$ - dash-dot-dotted lines, and $750 \times 4500$ - long-dashed lines. The results are shown for $G r=10^{4}, S c=10^{3}, M=10^{-7}$, $A=-0.5$, and $C n=10^{-4}$. (a) The vertical position of the droplet, (b) the volume of the droplet, (c) the speed of the droplet, (d) the total kinetic energy of the mixture in the computational domain, (e) the interfacial thickness, and (f) the surface tension coefficient.

Thus, for our estimations we take the density and viscosity of water, and the value of the diffusion coefficient that would be typical for a water-based mixture,

$$
\rho_{1} \sim 10^{3} \frac{\mathrm{kg}}{\mathrm{m}^{3}}, \quad \eta \sim 10^{-3} \mathrm{~Pa} \cdot \mathrm{s}, \quad D \sim 10^{-9} \frac{\mathrm{m}^{2}}{\mathrm{~s}} .
$$

Two most influential parameters that allow adjustments of the Grashof number are the concentrations of the mixture components, as they determine the gravity contrast, $\phi$, and the initial size of the droplet, as this also sets the typical length scale, $L_{*}$. We assume that the initial radius of the rising droplet is small, $a_{0} \sim 10^{-3} m$ (the container's size, which is chosen as the typical length scale, is taken ten-times greater, $R=L_{*} \sim 10^{-2} \mathrm{~m}$.) We also assume a lower value of the density contrast, $\phi \sim 10^{-3}$. In this case, the typical values of the nondimensional parameters that define the classical model are

$$
S c \sim 10^{3}, G r \sim 10^{4} .
$$

The chosen droplet's size and especially the density contrast would in fact be quite small for an experimental work. 
The given estimation of the Grashof number would rather set the lower boundary of the Grashof number for the dynamics of (diluted) water-based binary mixtures. For other mixtures, although, e.g. with more viscous components, like a water bubble in glycerol/water mixture, or molasses (see Ref. 1), the values of the Grashof number can be lower.

One of the difficulties of the phase-field modelling is involvement of non-classical phenomenological parameters, $\alpha$, $\mu_{*}$, and $\varepsilon$. First, to model the evolution of a mixture within the phase-field approach, we accept the same values of the Grashof and Schmidt numbers, as in the classical approach.

The value of the Cahn number is primarily defined by the capillary constant, $\varepsilon$. This parameter, in particular, determines the equilibrium interface thickness, which is $\delta_{e q}=\sqrt{-C n / A}$. In our work, the calculations are fulfilled for $C n=10^{-4}$, when the interfacial boundary is sufficiently thin, much smaller than the droplet's size, although the numerical resolution of such a boundary remains doable.

The value of the Mach number defines the magnitude of the Korteweg force, setting the importance of the surface tension effects. We fulfil the calculations for different Mach numbers in order to reveal the role of the capillary forces in the overall droplet's dynamics.

The thermodynamic model of the mixture is determined by the parameter $A$. The considered binary mixture is given by the phase diagram with the upper consolute point (that is defined by $A=0$ ): if $A$ is positive then the mixture is homogeneous in equilibrium, and if $A$ is negative then the mixture may be either homogeneous or heterogeneous as determined by the overall mass balance (for the considered problem, however, owing to the fact that the droplet is small as compared with the size of the container, the state of thermodynamic equilibrium will be always homogeneous, except for $A=-0.5$ when the interfacial diffusion is (nearly) zero, as the initial concentrations of the mixture components already correspond to the equilibrium saturation levels). In our work we perform the calculations for several different values of parameter $A$.

Let us also summarise the numerical values of the scales of the velocity and time, and surface tension coefficient, that would be needed for the analysis of the results,

$$
u_{*} \sim 10^{-4} \mathrm{~m} / \mathrm{s}, \tau_{*} \sim 10^{2} s, \sigma_{*}=\rho_{1} u_{*}^{2} L_{*} \sim 10^{-7} \mathrm{~N} / \mathrm{m} .
$$

The motion of an inclusion in an ambient liquid is frequently characterised by the Reynolds number that is defined on the inclusion's size,

$$
\operatorname{Re}=\frac{\rho_{*} u_{*} a_{0}}{\eta_{*}} \sim 0.1
$$

Lower value of the Reynolds number corresponds to a slowly moving inclusion that tend to preserve its initial spherical shape.

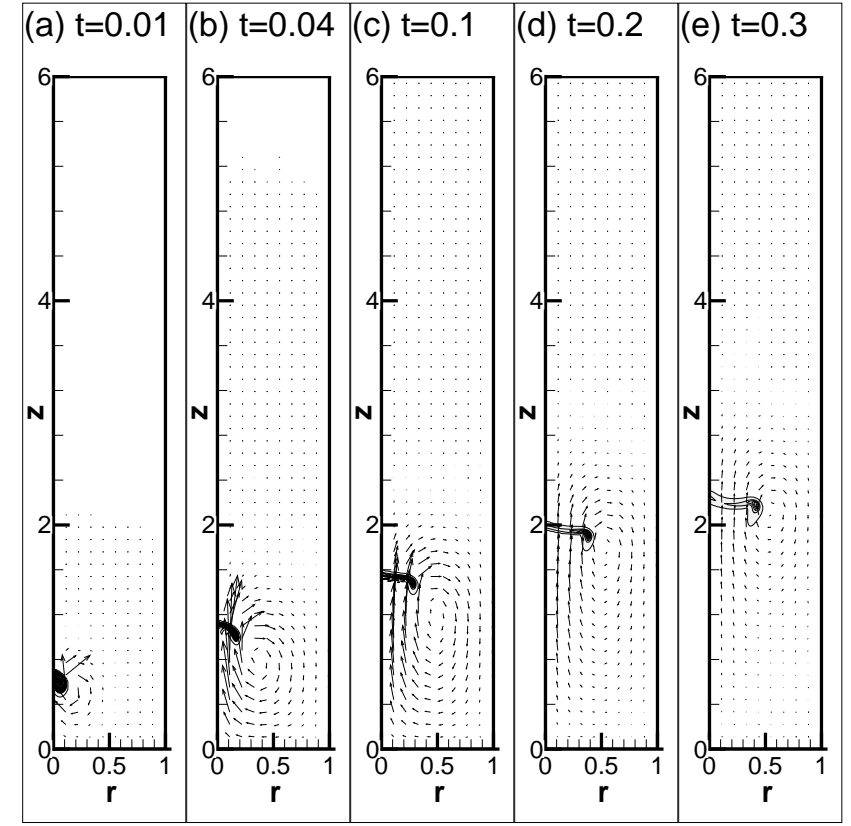

FIG. 2. The snapshots of the concentration and velocity fields. The results are obtained with the use of the classical approach, for $G r=$ $10^{4}$ and $S c=10^{3}$; the numerical resolution is $500 \times 3000$ grid-points. The snapshots are shown for the time moments, (a) $t=0.01$, (b) $t=0.04$, (c) $t=0.1$, (d) $t=0.2$, and (e) $t=0.3$.

\section{RESULTS}

\section{A. Classical approach}

Figure 2 depicts the flow fields for a rising droplet that is modelled using the classical approach. One can observe a very rapid disintegration of the droplet, so it becomes completely invisible at $t \sim 1$. By the moment of its disappearance $(\sim 1 \mathrm{~min})$ the droplet travels a relatively short path $(<3 \mathrm{~cm}$ in dimensional units). The Schmidt number for this calculation is sufficiently high, which sets a very large value of the diffusion time, $\tau_{d} \sim S c \sim 1000$, and thus the visible disappearance of a droplet should be primarily explained by its disintegration (spreading over the computational domain), assisted by convective motion.

The flattening of the droplet during its rise (and formation of the ring) resembles the experimental observations reported in Refs. 1 and 10. The initial dynamics of the droplet, including the intrusion of the ambient fluid from behind, also agrees with the earlier modelling results reported in Refs. 11 and 12.

We also performed the simulations at different values of the Grashof numbers. At higher Grashof numbers, the dispersion of the droplet occurs even faster (see figure 3). At $G r=10^{3}$ (see figure 4), the droplet's rise occurs much slower, although, at the same time, the droplet manages to travel a greater distance before its disappearance. At this level of the Grashof number, the hydrodynamic motion induced by the moving droplet in the ambient liquid is not strong enough to break the droplet. At higher Grashof numbers the speed of 


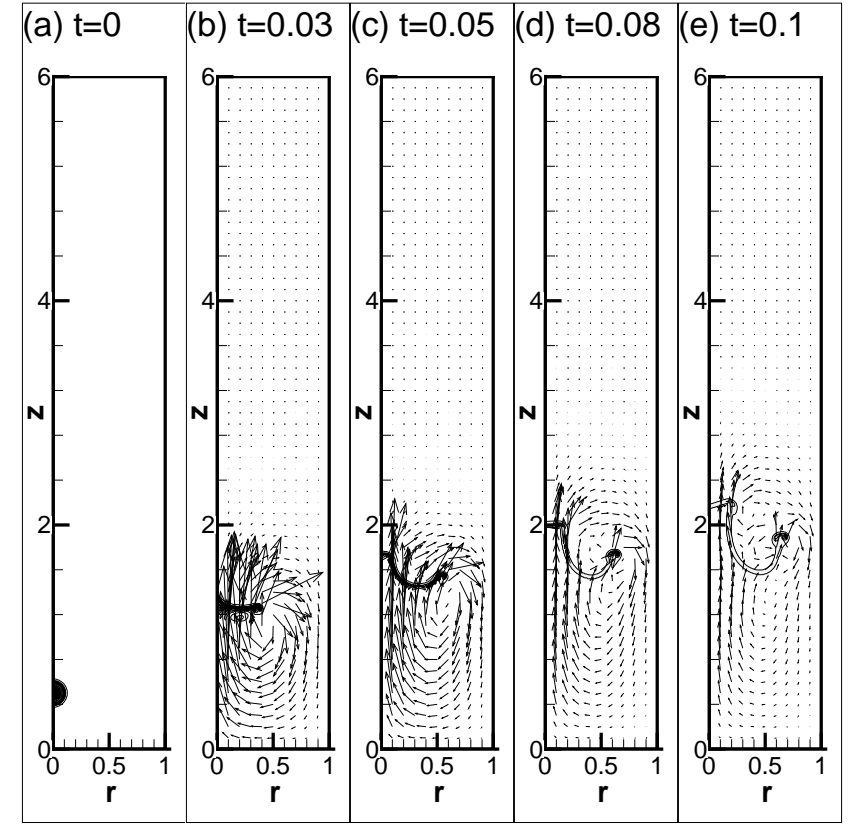

FIG. 3. The snapshots of the concentration and velocity fields at different time moments. The results are obtained using the classical model, for $G r=10^{5}$ and $S c=10^{3}$ and the resolution of $550 \times 3300$ grid nodes. The snapshots are shown for the time moments, (a) $t=0$, (b) $t=0.03$, (c) $t=0.05$, (d) $t=0.08$, and (e) $t=0.1$.

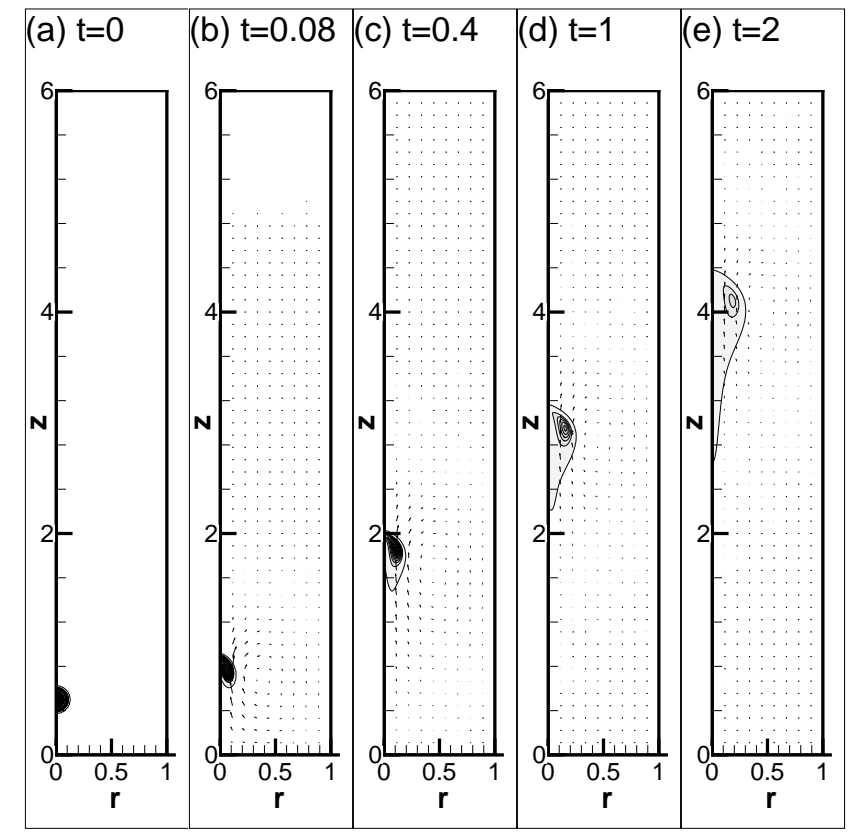

FIG. 4. The snapshots of the concentration and velocity fields at different time moments. The results are obtained with the use of the classical approach, for $G r=10^{3}$ and $S c=10^{3}$. The snapshots are shown for the time moments, (a) $t=0$, (b) $t=0.08$, (c) $t=0.4$, (d) $t=1$, and (e) $t=2$.

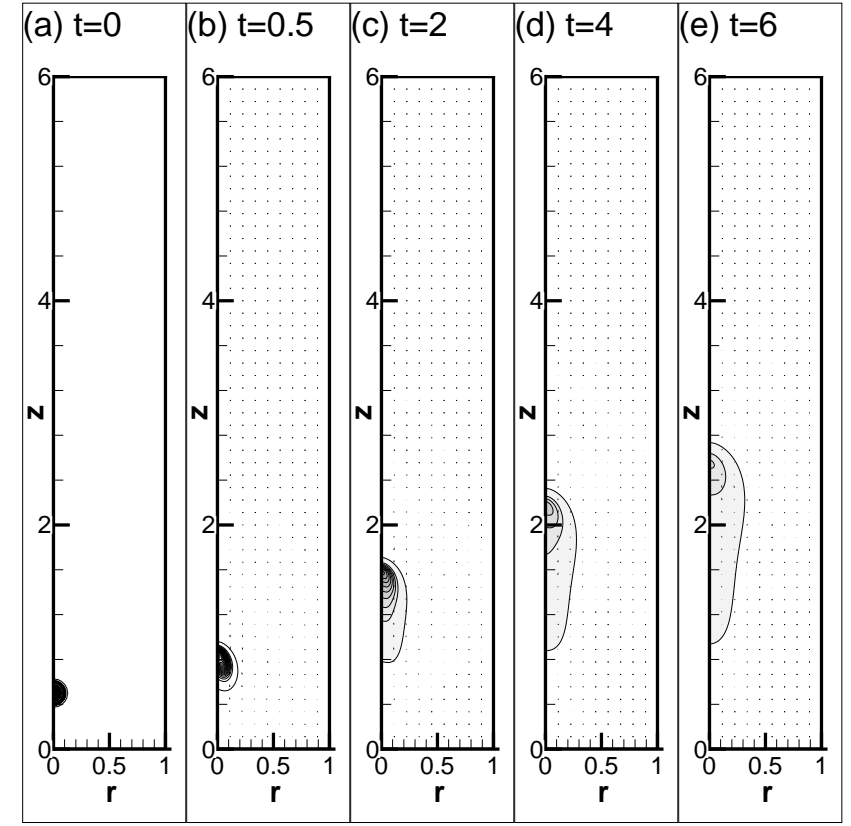

FIG. 5. The snapshots of the concentration and velocity fields at different time moments. The results are obtained with the use of the classical model, for $G r=10^{2}$ and $S c=10^{3}$. The snapshots are shown for the time moments, (a) $t=0$, (b) $t=0.5$, (c) $t=2$, (d) $t=4$, and (e) $t=6$.

the vortex formed at the droplet's side is comparable or even stronger than the droplet's speed of rise (especially, because the flattening of the droplet's shape substantially decelerates the droplet's rise), and this is not the case for lower Grashof numbers. At the lower Grashof numbers, the droplet's shape evolves to form a tail at the trailing edge, which closely resembles the modelling pictures by Koh \& Leal ${ }^{11}$ and Pozrikidis ${ }^{12}$. The calculations fulfilled at even lower Grashof numbers (see figure 5 for the results obtained for $G r=10^{2}$ ) show that at these Grashof numbers the diffusion effect becomes more pronounced, so the droplet's dynamics can be described as diffusive smearing (obviously, anisotropic smearing) rather than the hydrodynamic motion and dispersion. The effect of interfacial diffusion was not taken into account in the modelling works by Koh \& Leal ${ }^{11}$ and Pozrikidis ${ }^{12}$, and they did not report similar diffusion-driven evolution of the droplet's shape.

Some experimental images, e.g. those reported in work 1 , clearly show the prolong rises of droplets, that remain nearlyspherical. Moreover, the authors note that 'bubble appears to want to pull into a sphere even and especially at the instant of injection'. Our calculations allow us to conclude that miscible droplets, reported as absolutely unstable in Refs. 10-12, can in fact rise as a whole (without strong deformations), but only if they rise rather slowly, under lower Grashof numbers. Although, since the classical theory fully neglects the surface tension effects, it may never explain the experimental observations when a miscible 'bubble ... pulls into a sphere'. 


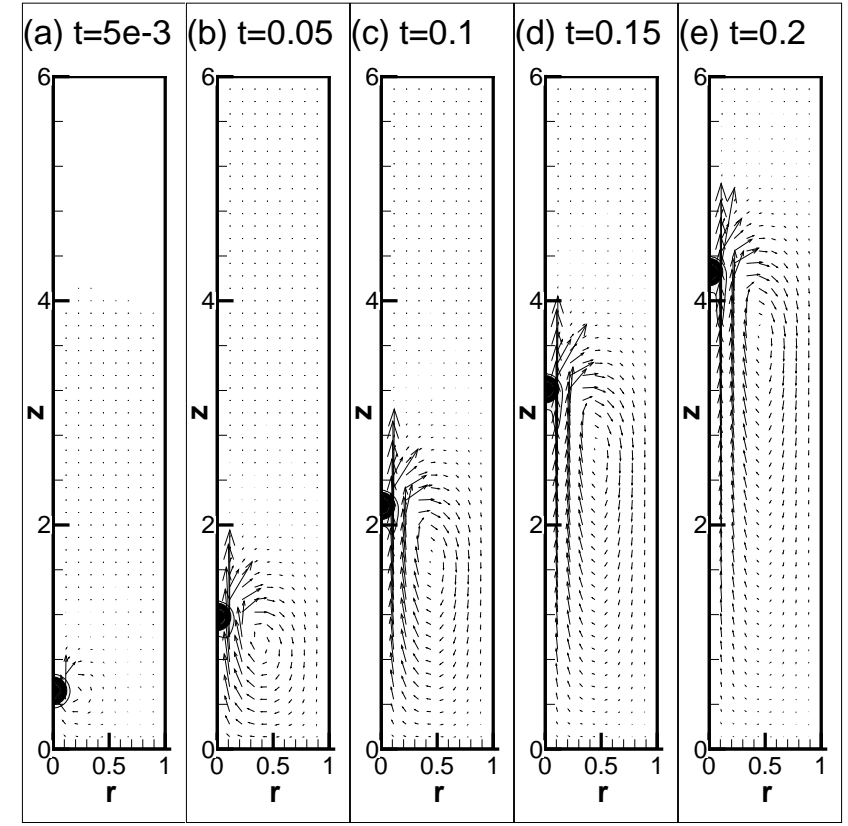

FIG. 6. The snapshots of the concentration and velocity fields at different time moments. The results are obtained with the use of the phase-field approach, for $G r=10^{4}, S c=10^{3}, A=-0.5, C n=10^{-4}$, and $M=10^{-7}$. The arrows that depict the velocity vectors are of the same length as in figure 2 . The snapshots are shown for the time moments, (a) $t=0.005$, (b) $t=0.05$, (c) $t=0.1$, (d) $t=0.15$, and (e) $t=0.2$.

\section{B. Phase-field modelling}

Figure 6 depicts the flow fields for a rising droplet that is modelled on the basis of the phase-field approach. One sees that the surface tension added to the interface keeps the droplet's shape spherical during the droplet's rise. Owing to lower drag, a spherical droplet rises much faster.

Figure 7 depicts the integral characteristics of a rising droplet. The results are shown for three different values of the Mach number (different levels of the surface tension forces). Figure 7a shows the vertical position of the droplet at different time moments. Figure $7 \mathrm{~b}$ shows the volume the droplet. The thick dashed line depicts the limiting value that corresponds to a spherical droplet with the radius of 0.1 . The volume of the droplet with interfaces endowed with stronger surface tension forces remains nearly constant during the entire rise. At lower values of the surface tension the interfacial diffusion becomes different from zero, which results in a slight shrinkage of the droplet.

After the period of an initial adjustment, the droplet starts to rise with the constant (terminal) speed. In the limiting case of a slowly rising spherical droplet, the terminal speed is determined by the Stokes' law (or the Hadamard-Rybczynski's law),

$$
U_{\infty}=\frac{4}{15 K_{1}} G r r_{0}^{2}
$$

This equation is written in assumption that the droplet's liq-

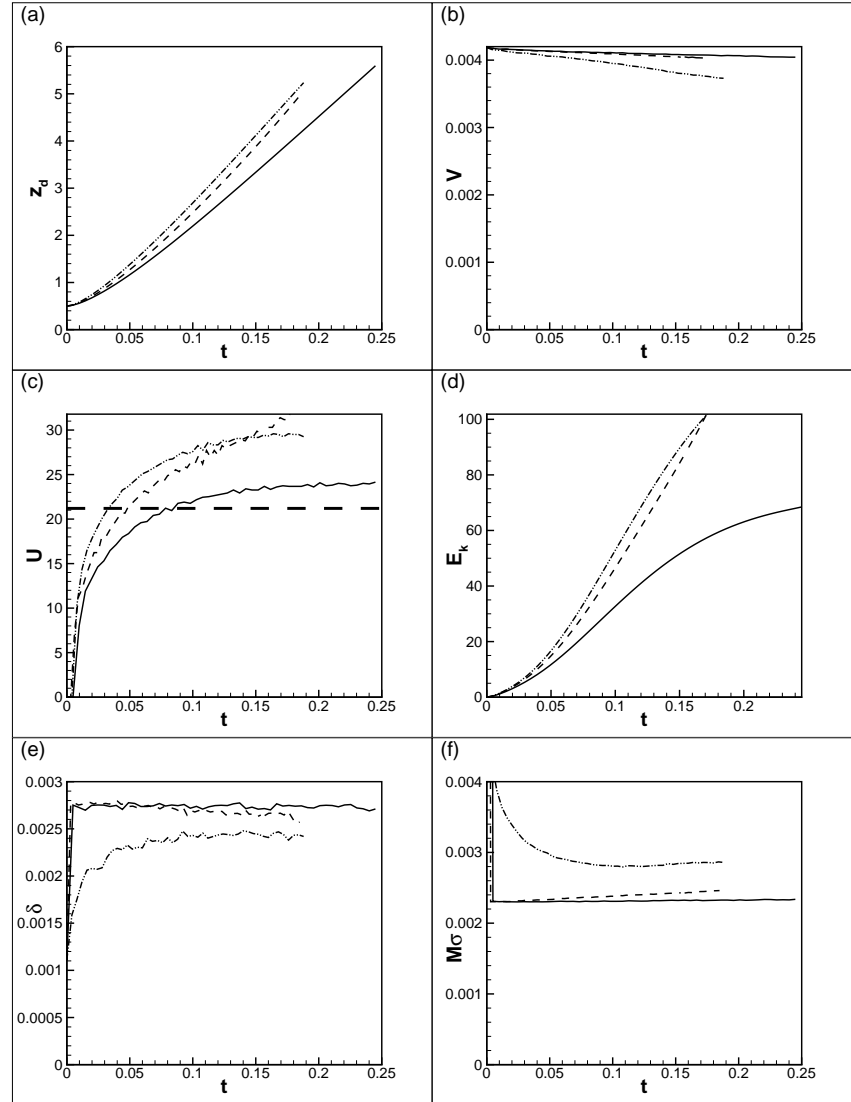

FIG. 7. (a) The vertical position, (b) volume, and (c) speed of a droplet; (d) the total kinetic energy of the fluid motion in the container; (e) the droplet's interfacial thickness; and (f) the coefficient of the surface tension. The data is obtained for $G r=10^{4}$, $S c=10^{3}, A=-0.5, C n=10^{-4}$, and three different Mach numbers, $M=10^{-5}$ (dash-dot-dotted line), $M=10^{-6}$ (dashed line), and $M=10^{-7}$ (solid line). The results are obtained with the numerical grid with $750 \times 4500$ grid points.

uid and ambient liquid have the same values of the viscosity coefficient. The correction coefficient $K_{1}$ takes into account the fact that the droplet rises in a tube of spherical cross section (i.e. it takes into account the influence of the container's walls on the droplet's speed). ${ }^{30}$ For the droplet of the radius $r_{0}=0.1$, the correction coefficient is $K=1.26$, giving the value of the terminal velocity, $U_{\infty}=21.2(\sim 2 \mathrm{~mm} / \mathrm{s}$ in the dimensional units). In figure $7 \mathrm{c}$, one sees that at lower Mach numbers, the calculated terminal speed of a droplet approaches the value predicted from formula (36).

The rising droplet engages into motion the fluid within the entire computational domain. The intensity of this motion is characterised by the total kinetic energy, $E_{k}=\frac{1}{2} \int_{V} u^{2} d V$, that is depicted in figure $7 \mathrm{~d}$. The kinetic energy associated with the rising droplet, $V U_{\infty}^{2} / 2 \approx 0.942$ is considerably smaller than the level of the resultant kinetic energy shown in figure $7 \mathrm{~d}$, indicating that in fact a significant part of the fluid domain is engaged into hydrodynamic motion.

Figure 7 e depicts the interfacial thicknesses calculated as the thickness of the zone for which $|C| \leq 0.05$. Figure $7 f$ 


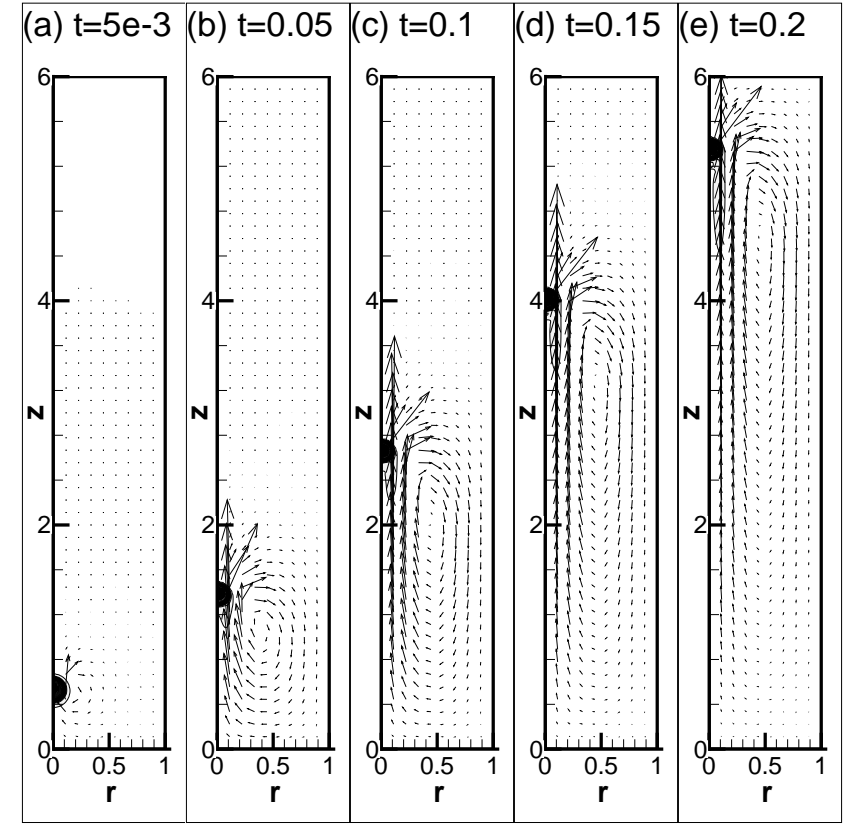

FIG. 8. The snapshots of the concentration and velocity fields at different time moments. The results are obtained with the use of the phase-field approach, for $G r=10^{4}, S c=10^{3}, A=-0.5, C n=10^{-4}$, and $M=10^{-5}$. The snapshots are shown for the time moments, (a) $t=0.005$, (b) $t=0.05$, (c) $t=0.1$, (d) $t=0.15$, and (e) $t=0.2$.

shows the value of the surface tension coefficient. Both the interface thickness and the surface tension coefficient associated with the interface remain nearly constant over the entire droplet's rise. By invoking the estimates (34) one may also understand that the dimensional value of the surface tension coefficient for the run with $M=10^{-7}$ is $\sigma_{d i m} \sim 10 \mathrm{~N} / \mathrm{m}$ and for $M=10^{-5}$ is $\sigma_{d i m} \sim 0.1 \mathrm{~N} / \mathrm{m}$.

The results shown in figures 6 and 7 depict the behaviour of a droplet which interface is endowed with very strong (in fact, non-physical) surface tension forces, when the droplet remains spherical (behaving like a solid particle). We are interested in the dynamics of the droplet with lower values of the surface tension, which can be adjusted by the Mach number.

Figures 8-11 show the shapes of the droplets with interfaces endowed with lower interfacial stresses. At $M=10^{-6}$ and $M=10^{-5}$ (see e.g. figure 8), there are no qualitative differences in the numerical results: the droplet continues to rise with a nearly-spherical shape. One may only notice that the speed of the droplet's speed becomes slightly higher (see figure 7c). Earlier, we already showed that the inclusions that are absorbed by the ambient liquid rise faster than they would rise in a non-absorbing liquid. ${ }^{21}$ This effect is explained by the action of the Korteweg force, similar to the Marangoni force that influences the rise of the bubbles with surfactants (see e.g. Refs. 31 and 32). At $M=10^{-4}$ (that should correspond to the surface tension coefficient of the or$\operatorname{der} \sigma_{\text {dim }} \sim 0.01 \mathrm{~N} / \mathrm{m}$, which is a typical value for an immiscible liquid/liquid interface), the droplet deforms into a ringlike shape (see figure 9). The stronger deformation of the droplet's shape also results in slowing down of the droplet's

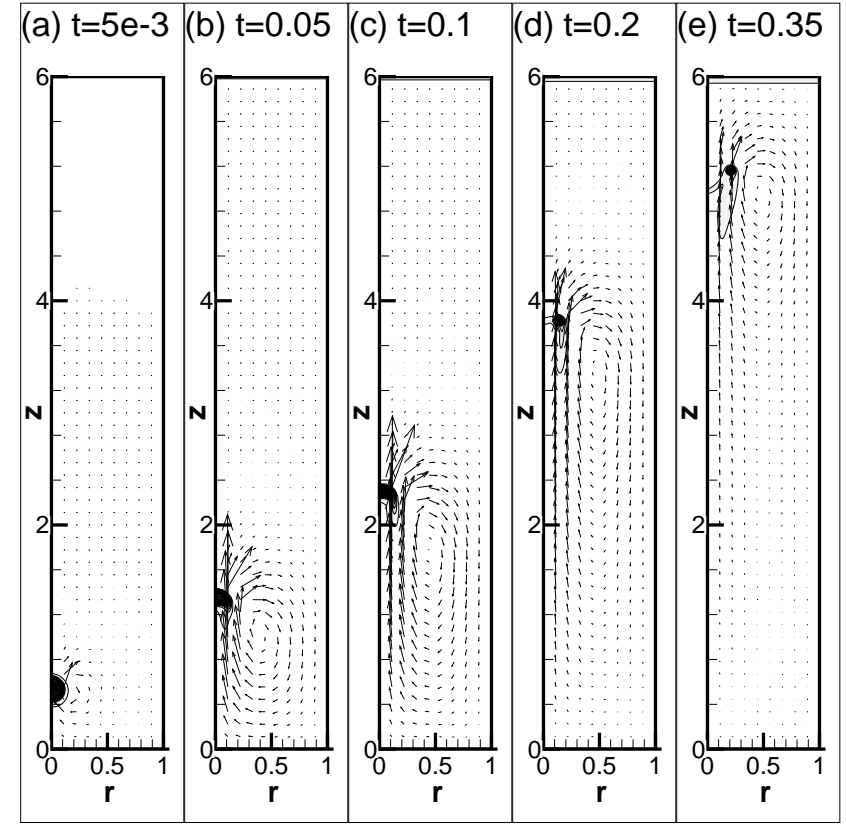

FIG. 9. The snapshots of the concentration and velocity fields at different time moments. The results are obtained with the use of the phase-field approach, for $G r=10^{4}, S c=10^{3}, A=-0.5, C n=10^{-4}$, and $M=10^{-4}$. The snapshots are shown for the time moments, (a) $t=0.005$, (b) $t=0.05$, (c) $t=0.1$, (d) $t=0.2$, and (e) $t=0.35$.

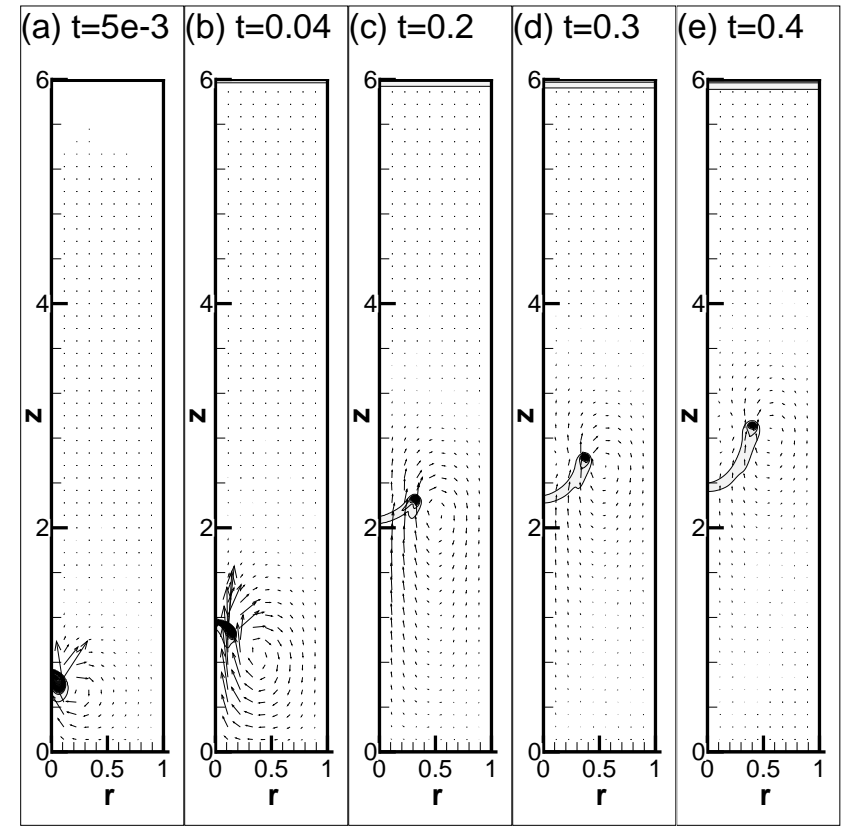

FIG. 10. The snapshots of the concentration and velocity fields at different time moments. The results are obtained with the use of the phase-field approach, for $G r=10^{4}, S c=10^{3}, A=-0.5, C n=10^{-4}$, and $M=10^{-3}$. The snapshots are shown for the time moments, (a) $t=0.005$, (b) $t=0.04$, (c) $t=0.2$, (d) $t=0.3$, and (e) $t=0.4$. 


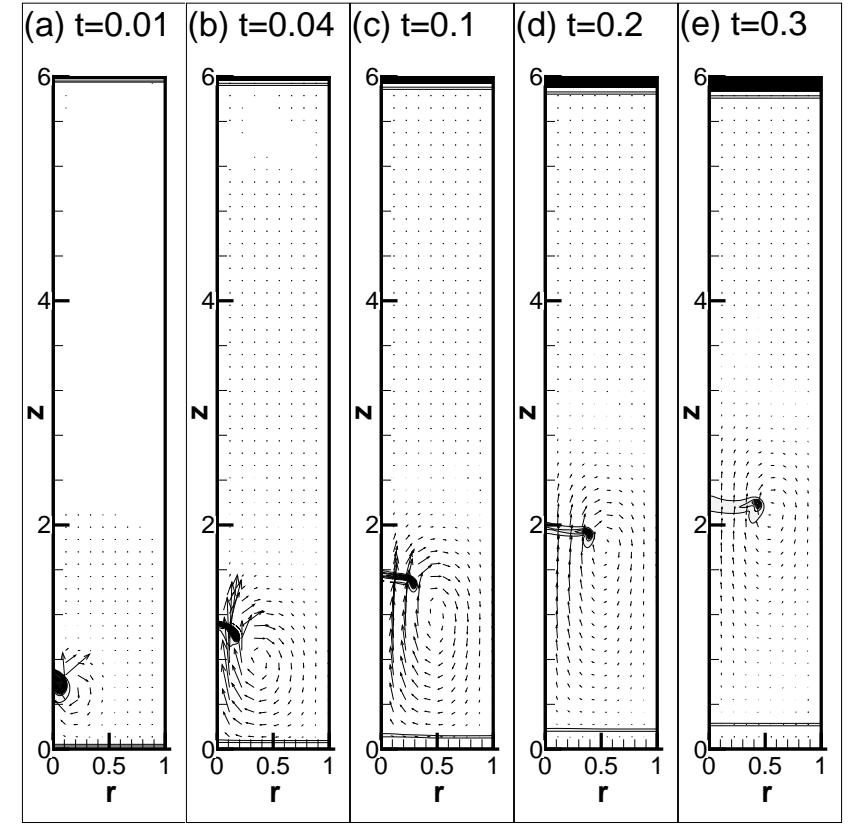

FIG. 11. The snapshots of the concentration and velocity fields at different time moments. The results are obtained with the use of the phase-field approach, for $G r=10^{4}, S c=10^{3}, A=-0.5, C n=10^{-4}$, and $M=10^{-1}$. The numerical resolution is $500 \times 3000$ grid-points. The snapshots are shown for the time moments, (a) $t=0.01$, (b) $t=0.04$, (c) $t=0.1$, (d) $t=0.2$, and (e) $t=0.3$.

rise. At $M=10^{-3}\left(\sigma_{d i m} \sim 10^{-3} \mathrm{~N} / \mathrm{m}\right)$, depicted in figure 10 , the droplet's dynamics already resembles the dynamics exhibited by the droplets with no surface tension (see figure 2). At even lower values of the Mach number (see figure 11 for $M=10^{-1}$ that should correspond to $\sigma_{\operatorname{dim}} \sim 10^{-5} \mathrm{~N} / \mathrm{m}$, i.e. the order normally assumed for miscible interfaces) the snapshots of the flow fields that characterise the droplet's rise quite closely reproduce the results shown in figure 2 . The differences at the flow field are only observed near the bottom and top walls of the container, where the phase-field results exhibit the development of the gravity-stratification in the concentration field due to effect of barodiffusion (which intensity is defined by $G r M$, and hence it increases at higher values of $M)$.

All the results shown above are obtained for $A=-0.5$. For the accepted thermodynamic model, this value of $A$ corresponds to the case of immiscible liquids, when the initial state of the mixture coincides with the state of thermodynamic equilibrium. The numerical results although show that in order to strictly eliminate the diffusion, it is additionally necessary to tend the Mach number to zero. At higher Mach numbers, the interfacial diffusion is low but still different from zero, which explains slight changes of the droplet's volume (see figure $7 b$ ).

Figure 12 demonstrates the results obtained for different values of parameter $A$. For $A>-0.5$ the shrinkage of a droplet due to the interfacial diffusion becomes more significant. The droplet's shrinkage results in slowing down of the droplet's rise (as the speed of the rise is proportional to the square of

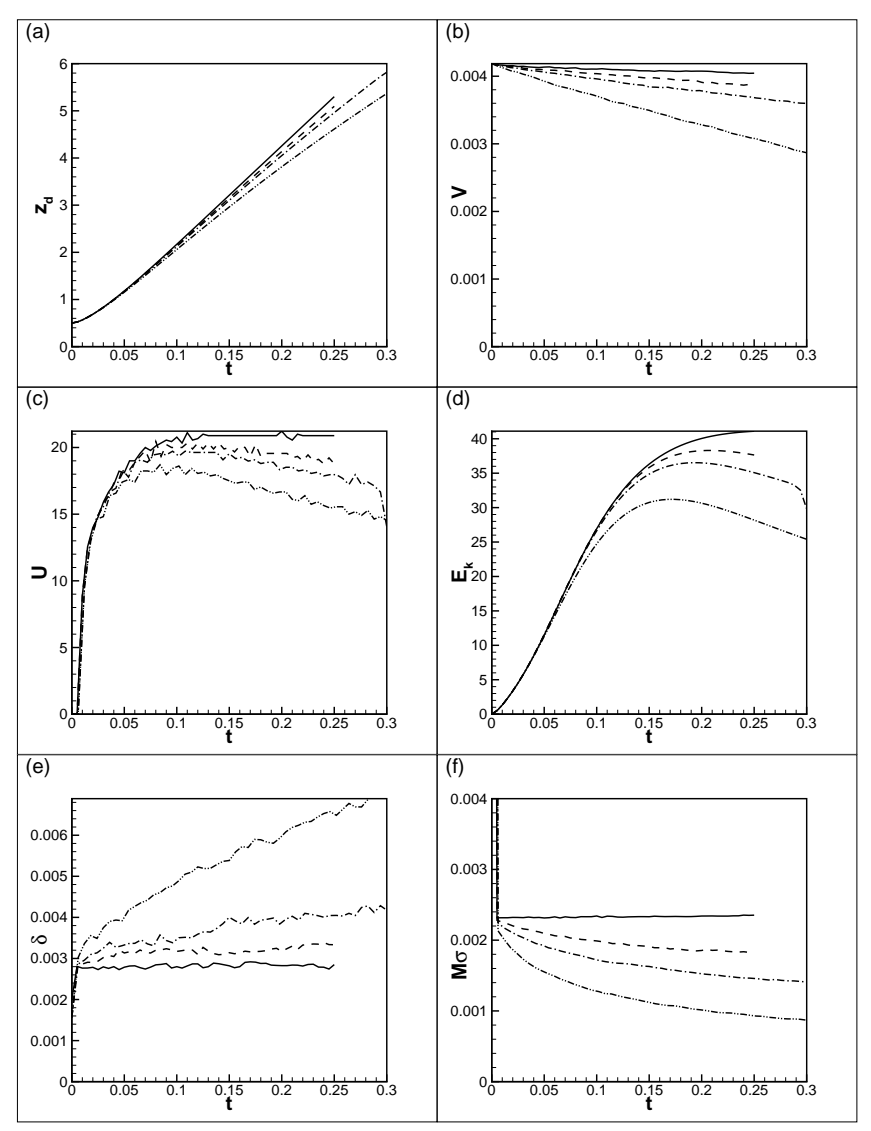

FIG. 12. (a) The vertical position, (b) volume, and (c) speed of a droplet; (d) the total kinetic energy of the fluid in the container; (e) the interfacial thickness; and (f) the coefficient of the surface tension. The data is obtained for $G r=10^{4}, S c=10^{3}, C n=10^{-4}, M=10^{-7}$ and for $A=-0.5$ (solid line), $A=-0.3$ (dashed line), $A=-0.1$ (dash-dotted line), and $A=0.5$ (dash-dot-dotted line).

the droplet's radius, see equation (36)). In addition, all calculations are started from the same initial state (defined by the same initial thickness of the interface). The temporal changes of the interfacial thickness are depicted in figure 12e. One sees that the thickness of the interface gradually grows. It should grow until either an equilibrium value is reached (for $A<0$, the equilibrium thickness of the interface is defined by $\sqrt{-C n / A}$ ) or indefinitely (for $A>0$ the state of thermodynamic equilibrium is always homogeneous). The temporal changes of the surface tension coefficient (depicted in figure 12f) follow the variations of the interface thickness, as it could be understood from equation (26).

The changes in the value of the surface tension over the period that is traced in our calculations are relatively small. For $M=10^{-7}$, no qualitative differences in the droplet's dynamics are observed for the calculations fulfilled with different $A$ (to observe significant changes, the calculations for a much longer container, much longer droplet's rise, would be needed). Although, for lower Mach numbers, when the droplet's trajectory remains limited to the modelled container's size, the calculations for different $A$ would already produce the visibly different data. For instance, figure 13 de- 


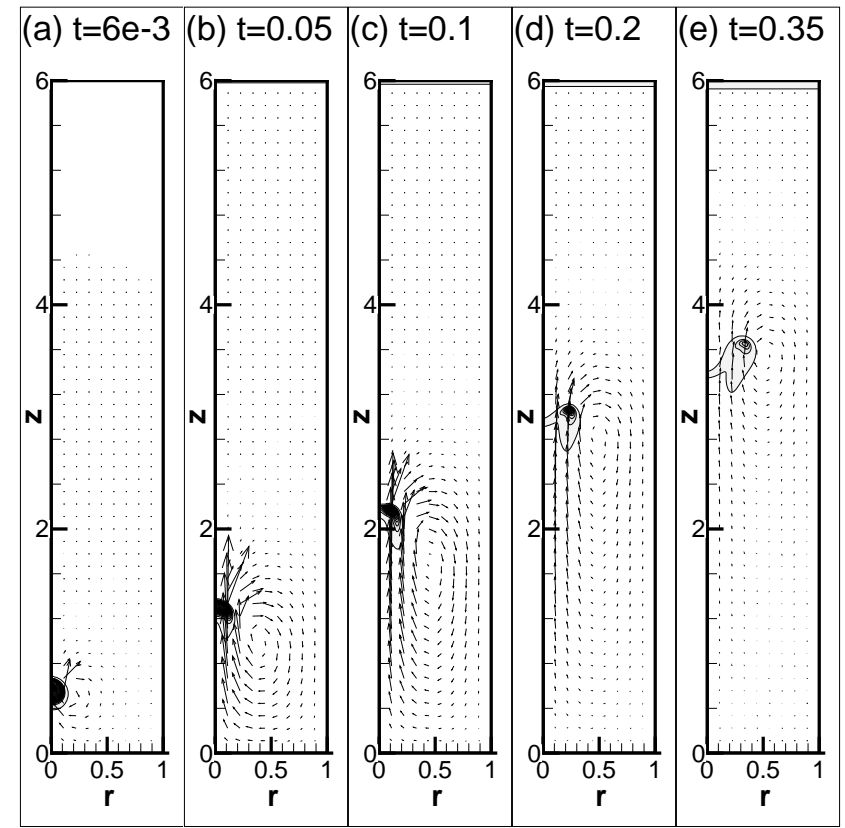

FIG. 13. The snapshots of the concentration and velocity fields at different time moments. The results are obtained with the use of the phase-field approach, for $G r=10^{4}, S c=10^{3}, A=0.5, C n=10^{-4}$, and $M=10^{-4}$. The snapshots are shown for the time moments, (a) $t=0.006$, (b) $t=0.05$, (c) $t=0.1$, (d) $t=0.2$, and (e) $t=0.35$.

picts the flow fields for the rising droplet obtained for $M=$ $10^{-4}$, as in figure 9 , but for $A=0.5$. The initial dynamics of the droplets for these two calculations are almost identical, but when the change in the surface tension becomes considerable $(t>0.1)$, the behaviour of the droplets becomes visibly different. Under higher temperatures (higher A) the droplet becomes more flattened, which slows downs its rise; the rate of interfacial diffusion is also increased.

\section{CONCLUSIONS}

We model the buoyancy-driven rise of a miscible droplet. Owing to lower diffusion coefficients in liquids, miscible droplets remain visible for quite long periods, during which they experience complex hydrodynamic evolutions. Miscible interfaces frequently exhibit the behaviour which can be only explained by involving the concept of the surface tension. For instance, it was noted that miscible droplets tend to have a spherical shape, which can be explained by the action of capillary forces. ${ }^{1}$ There were many attempts to measure the surface tension forces for miscible interfaces that produced generally quite low values, although, these values were still different from zero.

It was also shown that if the droplet's interface has no surface tension, then while moving it becomes absolutely unstable, rapidly dispersing within an ambient liquid. ${ }^{10-12}$ The dispersion of a droplet results in much faster visual disappearance of the droplet as compared with the expectations based on pure diffusion dynamics. It was also shown that the surface tension may suppress the development of the smaller perturbations, but still the perturbations with larger amplitude may lead to instability of the droplet's shape and to the droplet's dispersion.

In our study, we first modelled the droplet using the classical approach, completely neglecting the surface tension effects. In general, the obtained results agree with earlier theoretical studies of the motion of immiscible droplet with lower surface tension coefficients. ${ }^{11,12}$ We observe that the droplet rapidly disperses through a number of the already identified stages. Only for lower speeds of the droplet's rise, for lower Grashof numbers (for nearly density-matched mixtures), the shape of the rising droplets may resemble a sphere (at least such droplets do not brake into parts before their dissolution).

The classical modelling may not however explain why the droplet takes the spherical shape at the start of the evolution. Moreover, some experimental observations even report that miscible droplets may pull into shape of a sphere. ${ }^{1}$ These experimental observations motivated the second part of our work, where we develop the phase-field modelling of the rising droplet, taking into account the surface tension forces.

We carried out the numerical calculations for the fixed values of the Schmidt and Grashof numbers that would define the rise of a smaller droplet in a water-based binary mixture with nearly density-matched components, but for a wide range of the surface tension forces. For very high (non-physical) values of the surface tension coefficient, the droplet exhibits the behaviour of a solid particle, rising with preservation of a perfect spherical shape. At lower values of the surface tension coefficient (typical for immiscible liquid/liquid interfaces), the rising droplet deforms into shape of a ring. At even lower values of the surface tension coefficient, the dynamics of the droplet starts to resemble the results obtained with the help of the classical approach (for zero tension forces).

We also considered the rise of the droplets for the different 'mixture temperatures', which are set by the parameter $A$. This parameter sets the equilibrium state of the mixture, that may be heterogeneous (with e.g. the interface of the thickness $\sqrt{-C n / A})$ or homogeneous. By setting the initial value of the interface to be different from its equilibrium value we induce the adjustments of the interface thickness, which in turn results in dynamic adjustments of the surface tension coefficient associated with the interface. In that case, the system may be characterised by the higher value of the surface tension coefficient at the start of the droplet's rise, which explains the observed visible pulling of the droplet into the spherical shape at the initial moments (reported in work 1) and by the lower value of the surface tension to explain the features of the droplet's dynamics that are reproduced by the calculations that fully neglect the surface tension effects.

Finally, we conclude that disappearance of a miscible moving droplet (primarily due to dispersion) occurs on a short (hydrodynamic) time-scale. Over a short time period, the role of the surface tension on the droplet's dynamics is crucial, even more significant than the role of the interfacial diffusion. The dynamic changes of the surface tension coefficient strongly affect the overall dynamics of the droplet, so the droplet pulls into a spherical shape at the first instances, and behaves like 
a droplet with zero surface tension at the later period. These features of the dynamics of miscible interfaces can be successfully modelled on the basis of the phase-field approach.

\section{ACKNOWLEDGMENTS}

This research work is partially financially supported by the Russian Foundation for Basic Research (grant 18-01-00782) and by the UB RAS Program for Basic Research (project 1811-1-8). The authors also acknowledge the use of the IRIDIS High Performance Computing Facility, and associated support services at the University of Southampton, in the completion of this work.

${ }^{1}$ D. D. Joseph and Y. Y. Renardy, Fundamentals of Two-Fluid Dynamics. Part II: Lubricated Transport, Drops and Miscible Liquids (SpringerVerlag, New York, 1993).

${ }^{2}$ A. Vorobev, "Dissolution dynamics of miscible liquid/liquid interfaces," Current Opinion in Colloid and Interface Science 19, 300-308 (2019).

${ }^{3}$ S. E. May and J. V. Maher, "Capillary-wave relaxation for a meniscus between miscible liquids," Phys Rev Lett 67, 2013-1016 (1991).

${ }^{4}$ L. Lacaze, P. Guenoum, D. Beysens, M. Delsanti, P. Petitjeans, and P. Kurowski, "Transient surface tension in miscible liquids," Phys Rev E 82, 041606 (2010).

${ }^{5}$ J. A. Pojman, C. Whitmore, M. L. T. Liveri, R. Lombardo, J. Marszalek, R. Parker, and B. Zolotowski, "Evidence for the existence of an effective interfacial tension between miscible fluids: isobutyric acid-water and 1butanol-water in a spinning-drop tensiometer," Langmuir 22, 2569-2577 (2006).

${ }^{6}$ B. Zoltowski, Y. Chekanov, J. Masere, J. A. Pojman, and V. Volpert, "Evidence for the existence of an effective interfacial tension between miscible fluids. 2. dodecyl acrylate-poly(dodecyl acrylate) in a spinning drop tensiometer," Langmuir 23, 5522-5531 (2007).

${ }^{7}$ Y. A. Gaponenko, M. Torregrosa, V. Yasnou, A. Mialdun, and V. Shevtsova, "Dynamics of the interface between miscible liquids subjected to horizontal vibration,” J. Fluid Mech. 784, 342-372 (2015).

${ }^{8}$ D. Truzzolillo and L. Cipelletti, "Off-equilibrium surface tension in miscible fluids," Soft Matter 13, 13-21 (2017).

${ }^{9}$ A. Carbonaro, L. Cipelletti, and D. Truzzolillo, "Spinning drop dynamics in miscible and immiscible environments," Langmuir 35, 11330-11339 (2019).

${ }^{10}$ M. Kojima, E. J. Hinch, and A. Acrivos, "The formation and expansion of a toroidal drop moving in a viscous fluid," Phys Fluids 27, 19-32 (1984).

${ }^{11}$ C. J. Koh and L. G. Leal, "The stability of drop shapes for translation at zero reynolds number through a quiescent fluid," Phys Fluids A 1, 1309-1313 (1989).

${ }^{12}$ C. Pozrikidis, "The instability of a moving viscous drop," J. Fluid Mech. 210, 1-21 (1990).

${ }^{13}$ A. Vorobev and A. Boghi, "Phase-field modelling of a miscible system in spinning droplet tensiometer," J. Colloid Interface Sci. 482, 193-204 (2016).

${ }^{14}$ T. Lyubimova, A. Vorobev, and S. Prokopev, "Rayleigh-taylor instability of a miscible interface in a confined domain," Phys. Fluids 31, 014104 (2019).

${ }^{15}$ T. Zagvozkin, A. Vorobev, and T. Lyubimova, "Kelvin-helmholtz and holmboe instabilities of a diffusive interface between miscible phases," Phys. Rev E 100, 023103 (2019).

${ }^{16} \mathrm{~S}$. Prokopev, A. Vorobev, and T. Lyubimova, "Phase-field modeling of an immiscible liquid-liquid displacement in a capillary," Phys. Rev. E 99, 033113 (2019).

${ }^{17}$ A. Vorobev and T. Lyubimova, "Vibrational convection in a heterogeneous binary mixture. part 1. time-averaged equations," J. Fluid Mech. 870, 543562 (2019).

${ }^{18}$ A. Vorobev and T. Lyubimova, "Vibrational convection in a heterogeneous binary mixture. part 2. frozen waves,” J. Fluid Mech. 870, 563-594 (2019).

${ }^{19}$ H. Ding, P. Spelt, and C. Shu, "Diffuse interface model for incompressible two-phase flows with large density ratios," J. Comput. Phys. 226, 20782095 (2007).

${ }^{20}$ M. Tripathi, K. Sahu, and R. Govindarajan, "Why a falling drop does not in general behave like a rising bubble," Scient. Rep. 4, 4771 (2014).

${ }^{21}$ E. Khlebnikova and A. Vorobev, "Modelling of the rise and absorption of a fluid inclusion," Int. J. Heat Mass Trans. 125, 801-814 (2018).

${ }^{22}$ L. D. Landau and E. M. Lifshitz, Fluid Mechanics. Course of Theoretical Physics. Volume 6 (Elsevier, 1987).

${ }^{23}$ A. Vorobev, "Boussinesq approximation of the cahnhilliardnavierstokes equations,” Phys Rev E 82, 056312 (2010).

${ }^{24}$ L. D. Landau and E. M. Lifshitz, Statistical Physics. Course of Theoretical Physics. Volume 5 (Elsevier, 1980).

${ }^{25}$ M. Lappa and C. Piccolo, "Higher modes of the mixed buoyant-marangoni unstable convection originated from a droplet dissolving in a liquid/liquid system with miscibility gap," Phys. Fluids 16, 4262-4272 (2004).

${ }^{26}$ M. Lappa, "Oscillatory convective structures and solutal jets originated from discrete distributions of droplets in organic alloys with a miscibility gap," Phys. Fluids 18, 042105 (2006).

${ }^{27}$ F. B. Hicks, T. C. V. Vechten, and C. Franck, "Thermally perturbed barodiffusion in a binary liquid mixture," Phys Rev E 55, 4158-4164 (1997).

${ }^{28}$ K. Jamshidi-Ghaleh, M. T. Tavassoly, and N. Mansour, "Diffusion coefficient measurements of transparent liquid solutions using moire deflectometry," J Phys D Appl Phys 37, 1993-1997 (2004).

${ }^{29}$ E. V. Aitova, D. A. Bratsun, K. G. Kostarev, A. I. Mizev, and E. A. Mosheva, "Convective instability in a two-layer system of reacting fluids with concentration-dependent diffusion,' Journal of Applied Mechanics and Technical Physics 57, 345-358 (2016).

${ }^{30} \mathrm{~W}$. Haberman and R. Sayre, "Motion of rigid and fluid spheres in stationary and moving liquids inside cylindrical tubes," Tech. Rep. 1143 (David Taylor Model Basin, 1958).

${ }^{31}$ C. Ybert and J.-M. di Meglio, "Ascending air bubbles in protein solutions," Eur. Phys. J. B 4, 313-319 (1998).

${ }^{32} \mathrm{~S}$. Tasoglu, U. Demirci, and M. Muradoglu, "The effect of soluble surfactant on the transient motion of a buoyancy-driven bubble,' Phys. Fluids 20, 040805 (2008). 